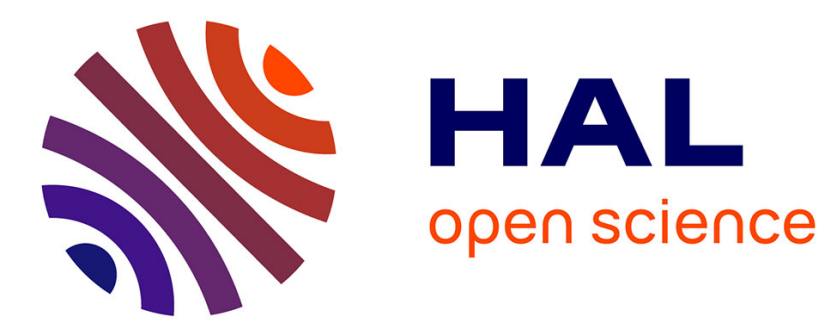

\title{
La pêche professionnelle dans les étangs Inférieurs de Basse Camargue: l'appropriation communale d'une frange du littoral méditerranéen (Note)
}

Carole Barthélemy

\section{- To cite this version:}

Carole Barthélemy. La pêche professionnelle dans les étangs Inférieurs de Basse Camargue: l'appropriation communale d'une frange du littoral méditerranéen (Note). Méditerranée : revue géographique des pays méditerranéens, 1999, 93 (4), pp.41 - 44. 10.3406/medit.1999.3129 hal-01756633

\section{HAL Id: hal-01756633 \\ https://hal.science/hal-01756633}

Submitted on 2 Apr 2018

HAL is a multi-disciplinary open access archive for the deposit and dissemination of scientific research documents, whether they are published or not. The documents may come from teaching and research institutions in France or abroad, or from public or private research centers.
L'archive ouverte pluridisciplinaire HAL, est destinée au dépôt et à la diffusion de documents scientifiques de niveau recherche, publiés ou non, émanant des établissements d'enseignement et de recherche français ou étrangers, des laboratoires publics ou privés. 


\section{La pêche professionnelle dans les étangs Inférieurs de Basse} Camargue : l'appropriation communale d'une frange du littoral méditerranéen (Note)

Carole Barthélémy

\section{Citer ce document / Cite this document :}

Barthélémy Carole. La pêche professionnelle dans les étangs Inférieurs de Basse Camargue : I'appropriation communale d'une frange du littoral méditerranéen (Note). In: Méditerranée, tome 93, 4-1999. Dynamiques naturelles et gestion des espaces littoraux. pp. 41-44;

doi : 10.3406/medit.1999.3129

http://www.persee.fr/doc/medit_0025-8296_1999_num_93_4_3129

Document généré le 13/06/2016 


\title{
La pêche professionnelle dans les étangs Inférieurs
}

\author{
de Basse Camargue : I'appropriation communale
}

\author{
d'une frange du littoral méditerranéen
}

Carole BARTHÉLÉMY*

La digue à la mer constitue un ouvrage essentiel dans la gestion du littoral camarguais. Édifiée en 1857 , elle isole une partie des étangs côtiers du milieu marin et s'étend à l'est de la commune des Saintes-Maries-de-la-Mer jusqu'au site de Beauduc, entre les deux bras du Rhône (fig. 1). Les communications entre la mer et les étangs ne s'établissent qu'au niveau de certains pertuis qui, équipés de martellières, permettent les passages d'eau douce ou d'eau salée. Cet équipement fait partie des aménagements présents sur le delta camarguais qui ont pour fonction de réguler les échanges hydrauliques. Ces endiguements (digue à la mer, digues du Rhône) ont été réalisés dans l'optique d'une exploitation du territoire débutée depuis la seconde moitié du XIX siècle et entièrement destinée à la mise en valeur agricole du delta. Ainsi, un sol rentable doit être dépourvu de toute salinité. La gestion adéquate sur ces terres deltaïques consiste donc à irriguer massivement par le fleuve et à éviter toute intrusion d'eau de mer (Picon, 1988). La construction de la digue à la mer s'inscrit dans cet objectif de dessalement des sols largement soutenue par les grands propriétaires agricoles locaux (PAILHÈs, 1999). Un syndicat est alors mis en place pour gérer les ouvertures et les fermetures des martellières et pour veiller à leur entretien.

Lorsque le sociologue observe la gestion de cet équipement aujourd'hui, il est frappé par le manque d'informations la concernant. Le syndicat de la digue à la mer n'existe plus (remplacé depuis 1996 par le SYDRHEMER, syndicat intercommunal de gestion des digues du Rhône et de la mer), les archives locales le concernant sont indisponibles et les entretiens auprès des divers intéressés (propriétaires agricoles, élus locaux, protecteurs de la nature...) apportent peu de précisions. La seule référence clairement explicitée est «la règle des trois 20 » qui stipule que le niveau de l'eau dans l'étang du Vaccarès doit être compris entre $+0,20$ et $-0,20 \mathrm{NGF}$ (mesure/niveau de la mer) et le taux de salinité doit se stabiliser autour de $0,20 \mathrm{gr} / \mathrm{litres}$. Ainsi peut être maintenue une certaine richesse biologique des milieux saumâtres tout en tenant compte des intérêts divergents des utilisateurs de l'eau. Les travaux de B. PICON ont montré comment cette gestion de l'eau est à l'origine de conflits sociaux en Camargue. L'agriculture, ayant besoin d'eau douce pour l'irrigation de cultures céréalières, s'est opposée dès le $\mathrm{XIX}^{\mathrm{e}}$ siècle à l'industrie salinière qui ne pouvait se développer que sur des terres salées. Les protecteurs de la nature à travers l'élaboration d'une Réserve nationale de Camargue ont joué un rôle intermédiaire entre ces deux opposants : "Ainsi, dans un quatrième temps, au début de ce siècle se met en place en Camargue une réserve intégrale de nature, résultante de la gestion hydraulique conflictuelle des agriculteurs et des saliniers»(PICON, 1996). L'importance accordée par ces trois groupes sociaux dominants au fonctionnement des pertuis qui régulent les sorties d'eau douce et les entrées d'eau salée prend alors tout son sens. Aujourd'hui, l'industrie salinière s'est déplacée vers l'est du delta, mais les

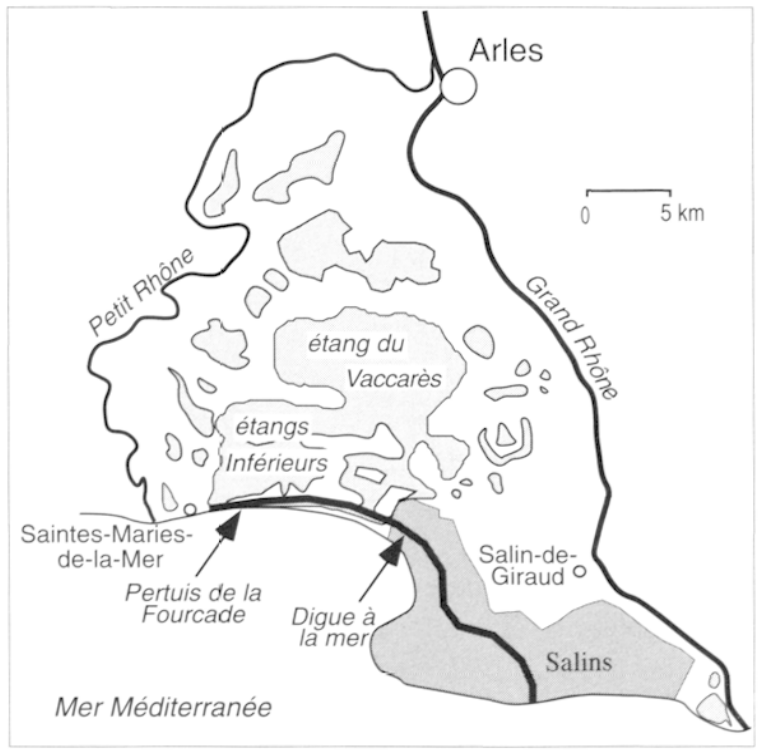

FIG. 1 - LA CAMARGUE : LOCALISATION DE LA DIGUE À LA MER

* Laboratoire DESMID (Dynamique écologique et sociale en milieu deltaïque) - ESA CNRS 5023, Arles. 
discordances autour de la gestion de l'eau perdurent entre les agriculteurs et les protecteurs de la nature. D'autres acteurs socioprofessionnels sont aussi concernés par cette gestion, notamment les pêcheurs professionnels qui exploitent les étangs Inférieurs de Basse Camargue. Ces étangs sont bordés par la digue à la mer et l'entrée d'eau de mer est capitale pour leur alevinage. L'activité de pêche professionnelle est donc aussi liée à la gestion hydraulique du delta. L'objet de cet article est d'analyser les pratiques des pêcheurs professionnels sur les étangs Inférieurs afin de comprendre les objectifs de gestion qu'ils aimeraient appliquer sur cet espace pour optimiser leurs prises. Mais, ces objectifs ne peuvent être abordés que dans le cadre d'une gestion collective de l'eau et il s'agira donc d'étudier la position de ces pêcheurs professionnels par rapport aux autres utilisateurs du delta camarguais.

\section{Les pêcheurs saintois et les étangs Inférieurs : une appropriation locale tardive et revendiquée}

Les étangs Inférieurs sont composés de l'étang du Malagroy et de l'étang de l'Impérial qui s'étendent sur 2800 hectares. Cet ensemble hydraulique dépend de l'étang du Vaccarès au nord, centre névralgique du delta et au sud, il communique avec la mer. Jusqu'en 1967, ces étangs appartenaient à la Compagnie des salins du Midi et étaient gérés par la Réserve nationale de Camargue. À cette date, l'État a exproprié la société salinière et confié ces étangs au Conseil général des Bouches-du-Rhône. Instaurée en «Réserve départementale», leur gestion et notamment celle de la pêche a alors été confiée à la mairie des Saintes-Maries-de-laMer, commune sur laquelle ils s'étendent. Les pêcheurs aujourd'hui retraités, racontent s'être battus pour l'obtention de cette zone de pêche qui était exploitée par des pêcheurs provenant pour l'essentiel du Languedoc-Roussillon. La pêche dans les étangs représentait pour les Saintois un complément non négligeable à la pêche en mer. Ainsi, depuis plus de trente ans, celle-ci est exclusivement réservée aux pêcheurs professionnels maritimes résidents de la commune des Saintes-Maries-de-la-Mer.

Les étangs Inférieurs sont divisés en deux espaces socialement marqués. L'étang du Malagroy au nord est essentiellement exploité par les jeunes pêcheurs professionnels (de 25 à 50 ans) et l'étang de l'Impérial au sud, plus proche du village, est réservé aux retraités de la Marine ; la commune leur octroie des postes de pêche. Le statut de Réserve départementale interdit l'utilisation de moteur, donc les pêcheurs utilisent une «partègue», longue perche de bois sur laquelle ils s'appuient pour avancer. Ce mode de déplacement distingue ainsi les vieux pêcheurs à qui l'on concède les postes les plus près. Ce partage entre les pêcheurs en activité et les retraités repose sur une volonté politique locale : "Il y a toujours eu des retraités qui pêchaient. C'est quelque chose qui doit être naturel. À l'origine, le village était un village de pêcheurs. Le maire était obligé de nous écouter sinon ça n'allait pas. L'ancien maire, son père était pêcheur. Donc, il est né làdedans» (entretien avec un retraité de la marine).

Divers arrêtés municipaux montrent que l'exercice de la pêche sur les étangs est remanié après chaque élection municipale. Les élus locaux se doivent de répondre aux attentes de cette corporation professionnelle qui légitime ses revendications sur leur forte représentation au sein du village. Cette représentation repose plus sur la pêche comme une activité fondatrice de l'identité villageoise que sur l'apport économique de cette activité (très largement dépassé par les activités touristiques) ou sur le nombre effectif de pêcheurs professionnels.

\section{L'exercice de la pêche sur les étangs : les savoir-faire à l'épreuve de l'aménagement}

La pêche sur les étangs de Basse Camargue est caractéristique de la pêche pratiquée sur les étangs du littoral méditerranéen (APKARIAN, 1996 ; Dufour, 1996 ; GiovanNoni, 1995 ; REY, 1996). Nécessitant de petites embarcations, elle repose sur l'utilisation de systèmes de nasses appelées «trabaques». De la berge est tendu un long filet, «la paradière», fixé au sol par des piquets de bois où le poisson vient «taper». À l'extrémité de ce filet sont fixées trois nasses formant chacune un piège. Ce type d'engins est adapté à la capture de certaines espèces piscicoles. Ces étangs sont en effet d'une très grande richesse piscicole car ils fonctionnent comme des zones de nursery (BARDIN, 1997). L'anguille est l'espèce la plus convoitée car son prix de vente est stable et le circuit économique organisé. Des mareyeurs viennent directement au bord de l'étang avec des camions équipés de caissons à oxygène. Les anguilles sont essentiellement exportées vers l'Italie, la Belgique et la Hollande. D'autres espèces sont pêchées, le joël ou l'athérine, la crevette grise, les soles et les daurades et sont commercialisées auprès d'un mareyeur local ou des mareyeurs du Grau-du-Roi.

Comme toute activité liée à un milieu naturel, les pêcheurs développent un ensemble de connaissances et de savoir-faire pour optimiser la pêche et son rendement. Ces savoirs reposent sur l'appréhension d'un monde invisible (GIOvanNONI, 1995 ; JoRION, 1990). D'une part, les pêcheurs apprennent à se déplacer sur l'étang. La définition d'un poste où seront calées plusieurs trabaques correspond à une connaissance du fonds des étangs qui répertorie les lieux de passage du poisson. Ainsi se construit une carte mentale des fonds (Dufour, 1985) (fig. 2). Dans ces étangs, le niveau d'eau est faible $(0,5 \mathrm{~m})$ et il varie rapidement en fonction des vents. Le pêcheur va donc caler ses nasses en fonction des vents dominants. Un vent de nord-ouest «bat les eaux» et le poisson est poussé vers la mer; au contraire, un vent de sud-est remonte le poisson vers l'étang du Vaccarès. Les postes tiennent aussi compte du niveau de l'eau. Il faut ainsi éviter les «plans» ou «plages» où l'eau manque et les cuvettes où l'eau 
abonde. Les trabaques sont calées près du contour de l'étang au niveau des «pointes» ou des «reculs», autour des «radeaux», petits îlots de terre. Enfin, il y a les «gazes», passages d'eau favorables au déplacement des poissons. Pour départager les postes, une course est organisée à l'ouverture des étangs. Un garde municipal donne le départ et tous les pêcheurs partent d'un même point et celui qui atteint un poste le plus rapidement se l'approprie pour la saison de pêche. Ce procédé, pour le moins original, représente un système consensuel imaginé par la mairie et repose sur un quadrillage des étangs construit et appréhendé grâce aux connaissances communes à tous les pêcheurs et non grâce à une désignation des postes formelle et écrite.

Un autre ensemble de savoirs concernent les déplacements des poissons non plus au sein des étangs mais au niveau de leur communication avec la mer. En effet, par le pertuis de la Fourcade, s'effectuent les échanges susceptibles d'engendrer une dynamique piscicole. Ainsi, les pêcheurs préconisent l'ouverture d'une ou deux martellières de novembre à juin. Lorsque l'eau s'écoule, le poisson peut rentrer, mais le contraire est aussi espéré. En effet, lorsqu'il y a des tempêtes de sud-est, l'eau de l'entre-plage (l'emplain) établit une communication et le poisson peut atteindre les étangs. Selon les pêcheurs, l'anguille colonise ces étangs dès le mois de novembre. Pour les autres espèces, l'alevinage a lieu à partir de février et durant tout le printemps. Ce calendrier de l'alevinage repose sur des observations d'alevins à certaines périodes (ainsi, «la savonnade» correspond à une eau troublée par la présence d'alevins de crevettes grises) dans les étangs et au niveau des martellières. Les pêcheurs sont aussi attentifs au cycle de la lune. En période d'obscur, la pêche à l'anguille est favorisée car celle-ci se déplacerait plus facilement. Les pêcheurs préconisent alors la fermeture des martellières les nuits sans lune car ils pensent que les anguilles peuvent alors rejoindre la mer.

\section{Une gestion de la digue modérée pour les pêcheurs d'étangs}

Les pratiques d'alevinage reposent sur le mouvement des martellières essentiellement du pertuis de la Fourcade. Les pêcheurs voudraient que ces martellières soient ouvertes plus souvent, à la fin de l'automne, au printemps ou lors de vents de sud-est. L'écoulement alors instauré ne doit pas être trop rapide car les poissons ne peuvent pas remonter un contrecourant trop puissant. Les martellières doivent être fermées la nuit en début de saison pour éviter la fuite des anguilles. Les échanges hydrauliques préoccupent aussi les pêcheurs. Un niveau d'eau trop faible dans les étangs peut entraîner un surcroît de salinité et donc nuire au bon développement des poissons. Par contre, un niveau d'eau trop élevé provenant de l'étang du Vaccarès peut adoucir les étangs et ne plus attirer le poisson de mer. Le fonctionnement des martellières souhaité par les pêcheurs est modéré. Il doit veiller à équilibrer le niveau d'eau, ni trop haut, ni trop bas ; le

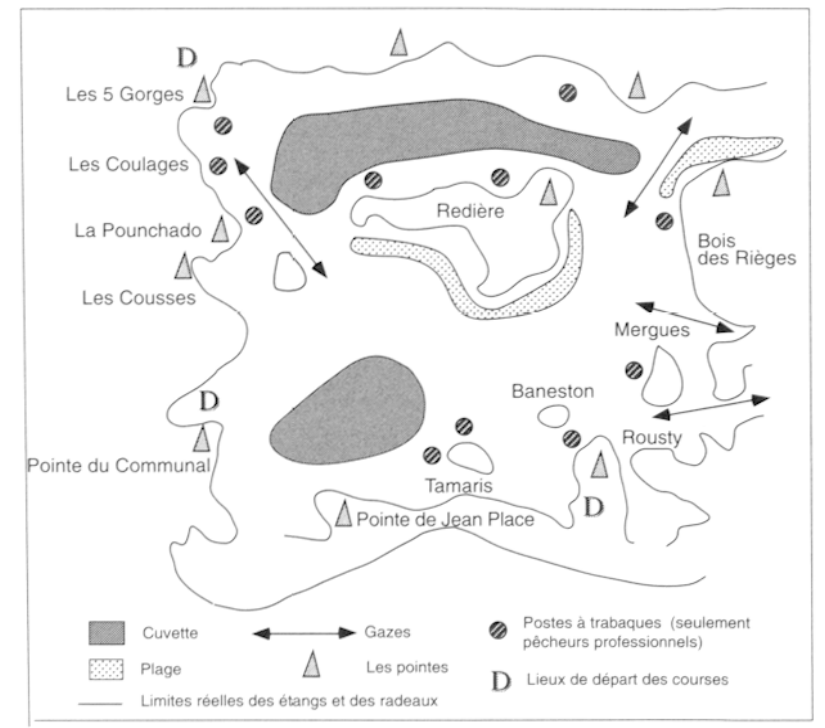

FIG. 2 - CARTE «MENTALE» DES FONDS DES ÉTANGS DE L'IMPÉRIAL ET DU MALAGROY

degré de salinité et le courant dans les vannes adéquates. Celles-ci doivent être ouvertes ou fermées en alternance selon les périodes. Ainsi, la digue à la mer n'est jamais remise en cause ; elle est au contraire totalemen intégrée à la perception du fonctionnement hydraulique des étangs. Cette gestion modérée correspond à la manière dont les pêcheurs se représentent les étangs, les fluctuations de niveau d'eau et les déplacements des poissons. Un juste équilibre entre l'eau, le sel et les mouvements piscicoles doit être selon eux préservé pour optimiser la pêcherie. Ce point de vue modéré doit aussi être analysé dans le contexte plus global de la gestion hydraulique du delta. Les pêcheurs saintois pensent être soumis aux décisions prises par les agriculteurs : "Il y a une paire de paysans qui ont les bras longs en Camargue et qui ne voudraient pas d'eau dans le Vaccarès. Ces gens, ils ne sont pas de chez nous. Ils arrivent de Paris... Ce sont toujours les paysans qui ont géré l'hydraulique en Camargue» (un pêcheur professionnel des étangs Inférieurs).

Ce sentiment d'être assujetti aux propriétaires locaux correspond à une réalité au vu de la nonreprésentation des pêcheurs au sein des instances décisionnelles (en 1998, un pêcheur a été élu au conseil d'administration du Parc naturel régional de Camargue). L'histoire conflictuelle entre les agriculteurs et les pêcheurs est cependant jonchée d'accords écrits (contractés en 1972, 1981 et 1988). Le dernier en date stipule que deux martellières seront ouvertes d'une période

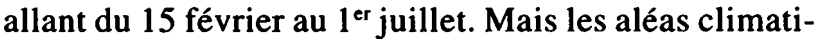
ques, qui entraînent des niveaux d'eau trop hauts ou trop faibles dans l'étang du Vaccarès, peuvent perturber ces directives. La gestion modérée souhaitée par les pêcheurs s'inscrit dans cette position de dominés dont ils se font tous l'écho. Ce sentiment contribue à ne s'exprimer que de manière discrète et relativement conforme à la gestion actuelle. Ce sont certes des opposants mais conscients de leur faiblesse. Le point de vue économique est ainsi très souvent énoncé stipulant que la pêche professionnelle est une activité moins productrice de richesse que l'agriculture ou le tourisme. 
4. Propriété, légitimation et protection : des modes d'appropriation des milieux naturels divergents

Jusqu'à présent, les pêcheurs d'étangs se sont donc plus adaptés à la gestion de la digue à la mer qu'ils n'ont pris part aux décisions en amont. Mais cela tend lentement à changer notamment avec l'avènement des crues de 1993 et de 1994 et la disparition du syndicat de la digue à la mer. Depuis, les décisions concernant les martellières sont prises au sein d'une commission spécifique au sein du Parc naturel régional de Camargue où siègent deux élus de la commune des SaintesMaries, dont un pêcheur professionnel ainsi que des représentants des propriétaires terriens et de la Réserve nationale de Camargue. Ainsi, en 1998, deux pêcheurs professionnels assistent aux concertations du Parc. De plus, la position de groupe social dominé doit être relativisée. La proximité du village avec le pertuis, et le fait que le garde-digue soit un employé de la commune des Saintes-Maries elle-même soucieuse des demandes des pêcheurs, laisse présager certaines marges de manœuvre.

L'analyse sociologique des pratiques de pêche et des représentations des pêcheurs des étangs Inférieurs nous conduit à deux conclusions. La première est d'ordre heuristique. L'étude des connaissances et des savoirfaire relatifs aux pratiques de pêche n'est pas seulement descriptive. Elle est complémentaire à une simple analyse des discours et des relations conflictuelles autour d'un espace. L'utilisation d'autres engins de pêche ou la recherche d'autres espèces piscicoles pourraient influer sur la gestion des étangs préconisée par les pêcheurs. De plus, l'analyse de cette corporation professionnelle considérée comme dominée permet d'observer certaines tendances concernant les conflits d'usage du littoral. Ainsi, l'opposition entre les pêcheurs professionnels et les agriculteurs repose sur deux modes d'appropriation de l'espace différents. Les agriculteurs sont d'abord propriétaires de leur exploitation mais les pêcheurs ne le sont pas. La propriété du sol et de la terre est longtemps restée un critère discriminant dans la gestion des milieux camarguais. Les agriculteurs justifient leur gestion hydraulique en mettant en avant ce sentiment d'appartenance au territoire camarguais généré par la propriété privée du sol et la mise en valeur des terres notamment en harmonie avec la protection de la nature (PICON, 1991). Au contraire, les pêcheurs justifient leur exploitation des étangs par leur ancrage au sein de la commune des Saintes-Maries et par l'image considérée comme traditionnelle du pêcheur en Camargue. Il n'est donc pas étonnant d'entendre les pêcheurs critiquer ces agriculteurs venus de Paris ou d'ailleurs ; ces derniers s'estimant plus «camarguais» que les propriétaires terriens. Cependant, les pêcheurs se réapproprient aussi l'idée de protection de la nature en considérant l'activité de pêche comme le garant de la bonne tenue des étangs. Ils surveillent, collaborent aux recherches scientifiques menées par les organismes de protection, ils se considèrent comme les gardiens de cet espace protégé. La vocation protectionniste dominante sur le territoire camarguais permet à chacun de légitimer sa place. Ainsi coexistent deux modes d'appropriation du territoire différents : l'un s'est établi sur la propriété $\mathrm{du}$ sol, revendiquant ainsi un pouvoir sur l'espace ; l'autre, au contraire, fort de sa mairie et de son maire, repose sur une gestion collective de l'eau et de la pêche. Cette dichotomie, qui jusqu'à présent a surtout servi l'agriculture, tend lentement à être remise en cause par les directives provenant des gestionnaires des espaces protégés. Leurs perceptions de la gestion hydraulique du delta qui intègrent plus facilement le fait de maintenir une certaine salinité dans les étangs contribuent à nuancer cette confrontation entre «les gens de la terre» et «les gens de l'eau».

\section{BIBLIOGRAPHIE}

Apkarian A., Vergès P., (1996).- Pêcheurs de Martigues : «les hommes d'un lieu». Terres Marines «Méditerranée, la pêche inventive», $\mathrm{n}^{\circ} 11$ avril-mai, p.17-22.

Allard P., Bardin O., Barthélémy C., Pailhès S., PICON B., (1999).- Modifications induites par les opérations de mise en valeur du delta du Rhône sur la dynamique des peuplements piscicoles de BasseCamargue, Rapport système écologiques et actions de l'homme, Programme CNRS environnement, vie et sociétés, $90 \mathrm{p}$.

Dufour A., (1985).- Entre «bouasque»et «bronde». Étude ethnologique de l'espace dans une commune du littoral provençal, Éd. du CNRS, Marseille, 211 p.

Dufour A., (1996).- «Petits métiers», grands savoirs, Terres Marines "Méditerranée, la pêche inventive», n'11 avril-mai, p.29-34.

Giovannoni V., (1995). - Les pêcheurs de l'étang de Thau. Écologie humaine et ethnologie des techniques, L'Harmattan, Paris, 283 p.
Jorion P., Delbos G., (1990).- La transmission des savoirs, La Maison des sciences de l'homme, $2^{\mathrm{e}}$ éd., Paris, 310 p.

Picon B., (1988).- L'espace et le temps en Camargue, Actes Sud, Arles, 230 p.

PiCon B., (1991).- La gestion hydraulique de la Camargue : une histoire de représentation in Chercheurs d'eau en Méditerranée, (sous la dir. de) C. AsPE, Éd. du Félin, p.65-73.

PiCon B., (1996).- De l'homme à la nature : l'exemple du delta du Rhône, in Impact de l'homme sur les milieux naturels, perceptions et mesures, Éditeurs scientifiques, Travaux de la Société d'écologie humaine, p.105-114.

REY H., (1996).- Pêches méditerranéennes : de l'économie informelle à la norme communautaire. Terres Marines "Méditerranée, la pêche inventive», $\mathrm{n}^{\circ} 11$ avril-mai, p.4-10. 